\title{
A novel tumor suppressor miRNA miR-520e contributes to suppression of hepatoma
}

\author{
Bo-an LI*
}

Acta Pharmacologica Sinica (2012) 33: 3-4; doi: 10.1038/aps.2011.198

$\mathrm{H}$ epatocellular carcinoma (HCC), one of the most common cancers worldwide, represents the third cause of cancer-related death ${ }^{[1]}$. Curative options for HCC are limited and exclusively available for patients carrying an early stage HCC. In advanced stages, traditional chemotherapy proved to be only marginally effective or even toxic. Thus, the identification of new treatment options is needed. New targets for nonconventional treatment will necessarily take advantage of progresses on the molecular pathogenesis of $\mathrm{HCC}^{[2]}$.

MicroRNAs (miRNAs) are short noncoding RNAs 19-25 nucleotides in length, which negatively regulate gene expression by pairing with complementary sequences within the 3'-untranslated regions (UTRs) of targeted transcripts at the post-transcriptional and/or translational level ${ }^{[3,4]}$. miRNAs expression is frequently deregulated in many types of human cancers, and plays critical roles in tumorigenesis, which functions either as tumor suppressors or as oncogenes. In the recent years, several studies have revealed that the expression of miRNAs is deregulated in human HCC in comparison with matched non-neoplastic tissue $^{[5]}$. More and more miRNAs involved in hepatocarcinogenesis were identified

State Key Laboratory of Cellular Stress Biology, School of Life Sciences, Xiamen University, Xiamen 361005, China

Correspondence: Prof Bo-an LI

(bali@xmu.edu.cn) in HCC tissues. Xu et al indicated that miR-195 may suppress tumorigenicity and regulate $G_{1} / S$ transition of $\mathrm{HCC}$ cells by repressing $\mathrm{Rb}-\mathrm{E} 2 \mathrm{~F}$ signaling involving targeting multiple molecules, such as cyclin D1, CDK6, and E2F3 ${ }^{[6]}$. Liang et al reported that miR-125b was able to suppress tumor growth in hepatic carcinogenesis through the suppression of oncogene LIN28B expression ${ }^{[7]}$. miR637 acts as a tumor suppressor, through the suppressive effect on HCC mediated by disruption of Stat3 activation ${ }^{[8]}$. miR-99a acts as a prospective prognosis predictor of HCC and potential tumor suppressor for HCC therapeutics, which induces cell cycle arrest by directly targeting IGF-1R and mTOR ${ }^{[9]}$. Thus, those miRNAs may function in a network in hepatocarcinogenesis.

In a recent issue of Oncogene, Zhang's group identified a novel tumor suppressor miRNA miR-520e in hepatoma cells. Zhang and colleagues demonstrated that the miR-520e was dramatically decreased in the examined hepatoma cells and clinical hepatocellular carcinoma (HCC) tissues ${ }^{[10]}$. In cancer, aberrant DNA hypermethylation of tumor suppressor genes, global genomic DNA hypomethylation and disruption of the histone modification patterns are the main epigenetic alterations, which have consequently been widely studied. Some miRNAs are downregulated in cancer and act as bona fide tumor suppressor genes, and this knowledge led to the proposal of the hypothesis that miRNAs could be silenced by epigenetic mechanisms ${ }^{[11]}$. The miR-520e gene is located in 19 q13.42 and is reported to be in chromosome 19 miRNA cluster (C19MC), the largest human miRNA gene cluster discovered so far. Their finding presented important information for the identification of potential mechanism of downregulation of miR-520e. The silencing of miR-520e was attributed to the methylation status of $\mathrm{CpG}$ islands, which are localized in the region of miR-520e promoter. Next, the paper used MTT assay, EdU assay, colony formation assay and analysis of tumorigenicity in nude mice to examine the potential role of miR520e in tumorigenesis. They found that miR-520e suppresses growth of hepatoma cells in vitro and in vivo. Then, they revealed that NF-kB-inducing kinase (NIK) was a direct target gene of miR$520 \mathrm{e}$. The NIK is a member of mitogenactivated protein kinase kinase kinase (MAP3K) family. NIK is usually constitutively and rapidly degraded by its TRAF3-mediated recruitment to TRAF2, which recruits cIAPs 1 and 2. NIK ubiquitination by these cIAPs promotes its proteosomal degradation. Stabilized NIK phosphorylates IKKa homodimers, which in turn phosphorylate p100 on its C-terminus, resulting in p100 ubiquitination and partial proteolytic processing by the proteosome to p52. The activated p52 then activates transcription when it associates with its binding partner (pri- 
marily RelB). Usually, NIK has a vital role in the alternative NF- $\mathrm{kB}$ activation pathway ${ }^{[12,13]}$. However, in this study they observed that NIK was able to activate NF-kB/p65 via ERK1/2 signaling. Taken together, the finding suggests that miR-520e suppresses hepatoma cell growth through NIK/p-ERK1/2/ $\mathrm{NF}-\mathrm{\kappa B}$ signaling pathway. Finally, they observed that the growth of tumor could be efficiently inhibited by direct injection with miR-520e in mice, suggesting that miR-520e might be a novel candidate for HCC therapy.

As the important roles of miRNAs in cancer are gradually revealed, their potential applications as useful and effective targets have generated great interest in cancer gene therapy strategies, as well as diagnosis, classification, prognosis and risk factor evaluations. In cell culture and xenograft mice models, the miRNA could cause tumor regression. One of the major problems of miRNA therapy is the delivery to only a restricted number of cells, which will certainly limit the therapeutic efficacy and provide only partial knock-down ${ }^{[14]}$. Thus, additional work needs to be carried out to improve the delivery of miRNA including novel chemical modifications of miRNA targeting molecules, development of novel devices for sustained delivery of therapeutics and engineering of novel viral vectors. The current large number of intense research and development activities in the area of miRNA therapy will further strengthen the possibilities of affecting modern medicine.

1 Parkin DM, Bray F, Ferlay J, Pisani P. Global cance statistics, 2002. CA Cancer J Clin 2005; 55: 74 108.

2 Gramantieri L, Fornari F, Callegari E, Sabbioni S, Lanza G, Croce CM, et al. MicroRNA involvement in hepatocellular carcinoma. J Cell Mol Med 2008; 12: 2189-204.

3 Bartel DP. MicroRNAs: target recognition and regulatory functions. Cell 2009; 136: 215-33.

4 Bartel DP, Chen CZ. Micromanagers of gene expression: the potentially widespread influence of metazoan microRNAs. Nat Rev Genet 2004; 5 : 396-400.

5 Murakami Y, Yasuda T, Saigo K, Urashima T, Toyoda $\mathrm{H}$, Okanoue $\mathrm{T}$, et al. Comprehensive analysis of microRNA expression patterns in hepatocellular carcinoma and non-tumorous tissues. Oncogene 2006; 25: 2537-45.

$6 \mathrm{Xu} \mathrm{T}$, Zhu Y, Xiong Y, Ge YY, Yun JP, Zhuang SM. MicroRNA-195 suppresses tumorigenicity and regulates $\mathrm{G}_{1} / \mathrm{S}$ transition of human hepatocellular carcinoma cells. Hepatology 2009; 50: 113-21.

7 Liang L, Wong CM, Ying Q, Fan DN, Huang S, Ding J, et al. MicroRNA-125b suppressesed human liver cancer cell proliferation and metastasis by directly targeting oncogene LIN28B2. Hepatology 2010; 52: 1731-40.

8 Zhang JF, He ML, Fu WM, Wang $\mathrm{H}$, Chen LZ, Zhu $X$, et al. Primate-specific microRNA-637 inhibits tumorigenesis in hepatocellular carcinoma by disrupting signal transducer and activator of transcription 3 signaling. Hepatology 2011; 54: 2137 48.

9 Li D, Liu X, Lin L, Hou J, Li N, Wang C, et al. MicroRNA-99a inhibits hepatocellular carcinoma growth and correlates with prognosis of patients with hepatocellular carcinoma. J Biol Chem 2011; 286: 36677-85.

10 Zhang S, Shan C, Kong G, Du Y, Ye L, Zhang X. MicroRNA-520e suppresses growth of hepatoma cells by targeting the NF-kappaB-inducing kinase (NIK). Oncogene 2011. doi: 10.1038/ onc.2011.523.

11 Lujambio A, Esteller M. CpG island hypermethylation of tumor suppressor microRNAs in human cancer. Cell Cycle 2007; 6: 1455-9.

12 Dhawan P, Richmond A. A novel NF-kappa B-inducing kinase-MAPK signaling pathway up-regulates NF-kappa B activity in melanoma cells. J Biol Chem 2002; 277: 7920-8.

13 Foehr ED, Bohuslav J, Chen LF, DeNoronha C, Geleziunas R, Lin X, et al. The NF-kappa B-inducing kinase induces PC12 cell differentiation and prevents apoptosis. J Biol Chem 2000; 275: 34021-4.

14 Davidson BL, Harper SO. Viral delivery of recombinant short hairpin RNAs. Methods Enzymol 2005; 392: 145-73. 